\title{
ANÁLISE DE PROCESSOS NO ENSINO DE GRADUAÇÃO: UMA ESTRATÉGIA DIDÁTICA BASEADA NO USO CONJUNTO DA SIMULAÇÃO COMPUTACIONALEDO PLANEJAMENTO E ANÁLISE DE EXPERIMENTOS
}

\section{PROCESS ANALYSIS IN UNDERGRADUATE COURSE : ONE TEACHING STRATEGY BASED ON THE CONJOINT USE OF COMPUTER SIMULATION AND DESIGN AND ANALYSIS OF EXPERIMENTS}

\author{
Noel Torres Júnior ${ }^{1}$; Júlia Zandomenico do Nascimento ${ }^{2}$; Guilherme Gonçalves de Souza ${ }^{3}$ \\ ${ }^{1}$ Universidade Federal de Minas Gerais - UFMG - Minas Gerais - Brasil \\ noelface@gmail.com / noel@face.ufmg.br \\ ${ }^{2}$ Universidade Federal de Minas Gerais -UFMG - Minas Gerais - Brasil \\ juliazn@globo.com \\ ${ }^{3}$ Universidade Federal de Minas Gerais - UFMG - Minas Gerais - Brasil \\ guilhermegoncalvesdesouza@gmail.com
}

\begin{abstract}
Resumo
Dada a centralidade do uso da simulação computacional de eventos discretos e da técnica de Planejamento e Análise de Experimentos para o desenho e melhoria dos processos existentes nas organizações, este trabalho aborda o ensino destas técnicas no curso de graduação. $O$ mesmo apresenta uma estratégia de ensino cuja ênfase recai na compreensão e análise do processo que será simulado e aperfeiçoado em contraste com abordagens existentes que privilegiam o conhecimento das rotinas e especificidades dos softwares dedicados a estas técnicas. A abordagem proposta é baseada nos trabalhos de Laguna e Marklund (2004), Hartvigsen (2005) e Mendenhall e Sincic(2012). A simulação computacional é feita por meio de planilhas eletrônicas utilizando-se o pacote SimQuick. $O$ uso do Planejamento e Análise de Experimentos para otimização dos processos é feito por ANOVA e por meio da Regressão Linear Múltipla. Um caso realizado por um grupo de alunos é apresentado como recurso didático para esclarecer a estratégia de ensino proposta.
\end{abstract}

Palavras-chave: análise de processos; simulação de eventos discretos; otimização.

\section{Introdução}

O desenvolvimento da informática conduziu a uma utilização generalizada de métodos de simulação e ferramentas em diversas áreas. Como exemplos deste fenômeno, pesquisadores, médicos e cirurgiões estão cada vez mais utilizando técnicas virtuais de operação antes de testar ou usá-las em pacientes reais; os simuladores são usados no treinamento de pilotos e no teste de manobras complicadas por navios ou aviões (BERENDS e ROMME, 1999). A simulação pode ser 
vista ainda como instrumento que auxilia na aprendizagem e na incorporação de inovações dentro das organizações, e se faz cada vez mais presente em diversas áreas e aplicações (SCHARAGE, 1999).

A despeito da importância desta técnica para as organizações, alguns fatores podem ser entendidos como obstáculos à maior disseminação e uso da simulação. Por exemplo, pode-se afirmar que muitos destes softwares apresentam certo nível de complexidade que demanda substancial conhecimento de seus comandos e bibliotecas, fazendo com que o instrutor e seus alunos dediquem um tempo considerável da disciplina ou do curso no entendimento do programa, em detrimento do maior conhecimento acerca do processo que deverá ser simulado (BORN e STÅHL, 2008). Além disto, deve se pesar o fato do software ter seus comandos e menus em uma língua estrangeira. Outro obstáculo consiste no elevado preço de aquisição desses programas.

A despeito destas restrições, algumas iniciativas se contrapõem a estes obstáculos, possibilitando uma melhor divulgação e ensino destas técnicas, uma vez que novas abordagens para ensino e uso da simulação de eventos discretos são propostas. Nestas, a simulação computacional pode ser realizada por meio de planilhas eletrônicas ou utilizando softwares mais amigáveis, simples e baratos (HILL, 2002). O enfoque maior fica na compreensão do processo que será simulado e no estudo das possibilidades de redesenho. A compreensão do software não ocupa mais o espaço central no ensino destas técnicas.

Em várias situações, apenas a simulação não é capaz de levar a uma compreensão do processo que indique o ponto ótimo de sua operação. Para tanto, torna-se necessário o uso de outros métodos que busquem este fim. Os autores Medina e Chwif (2007) colocam a existência de três tipos de estratégias no que tange à otimização. A primeira delas consiste na técnica da tentativa e erro que alterará aleatoriamente os valores das variáveis de modo a obter um conjunto de valores que "otimizem" sua medida de desempenho. A segunda consiste numa "análise de sensibilidade", alterando sistematicamente as variáveis e observando seus efeitos sobre o resultado. Esta poderá aplicar alguma técnica sistemática, como o Planejamento e Análise de Experimentos (Design of Experiments - DOE). O terceiro tipo aplicará diretamente um procedimento de Simulation Optimization. Este consiste na junção das ferramentas de simulação e otimização, em que a função objetivo, as restrições ou ambas são respostas que somente podem ser avaliadas pela simulação computacional.

A análise de processos pode ser melhor realizada por meio da simulação computacional de eventos discretos. Esta, por sua vez, poderá alcançar melhores resultados se utilizar algum método de otimização. Portanto, o uso conjunto destas duas técnicas proporcionará um grau de conhecimento maior sobre o processo que possibilitará a obtenção de melhorias significativas no seu desempenho (MEDINA e CHWIF, 2007). Diante da relevância destas técnicas, várias 
iniciativas voltadas para a melhoria de desempenho organizacional, conduzidas pelas organizações fazem uso das mesmas (PYZDEK e KELLER, 2011). Neste contexto, o ensino destas técnicas na formação de engenheiros de produção e gestores da área produtiva pode ser entendido como muito relevante.

Utilizando-se de uma abordagem apoiada no uso de planilhas eletrônicas e voltada para a compreensão do processo, o presente trabalho ilustra, por meio de um estudo de caso, uma estratégia de ensino destas duas técnicas. A abordagem proposta baseia-se, principalmente, nos trabalhos de Laguna \& Marklund (2004), Hartvigsen (2005) e Mendenhall \& Sincich (2012). Nesta abordagem, o uso de métodos e ferramentas para a análise e compreensão do processo a ser modelado são privilegiados. Nesta direção, a simulação é utilizada como uma ferramenta que apoia estas atividades. A mesma é feita por meio de planilhas eletrônicas utilizando-se o pacote SimQuick (HARTVIGSEN, 2005). O ensino do Planejamento e Análise de experimentos é feito por meio da analise de variância e por meio da regressão com do uso de planilhas eletrônicas. Para tanto, este trabalho descreve a importância da análise e da simulação de processos, e o uso de planejamentos de experimentos fatoriais. Posteriormente apresenta a estratégia de ensino construída dentro desta nova abordagem, e, finalmente, exibe um caso efetivado por um grupo de alunos, como recurso didático para esclarecer a estratégia de ensino proposta.

\section{Análise de processos e as técnicas de simulação e otimização}

As organizações podem ser entendidas como uma coleção de processos. Estes devem ser continuamente aperfeiçoados como uma estratégia para melhoria da qualidade e da produtividade do próprio negócio. Uma atividade essencial para que organização caminhe nesta direção é o conhecimento dos processos existentes e a proposição de melhorias ou redesenhos nos mesmos. Estas atividades dependem, em grande parte, do uso de métodos e técnicas de modelagem e da própria simulação computacional (LAGUNA e MARKLUND, 2004).

A modelagem dos processos pode ser feita por meio de modelos simbólicos que utilizam diversas técnicas de representação gráfica, tais como fluxogramas e o diagrama PERT, e a simulação computacional pode ser feita por meio de softwares dedicados.

\subsection{A simulação de eventos discretos}

A Simulação pode ser definida como a construção e manipulação operacional de um modelo, isto é, uma representação física ou simbólica de todos ou de alguns aspectos de um processo físico e ou social (BERENDS e ROMME, 1999).

Os sistemas reais apresentam maior complexidade que os modelos, pois estes primeiros mudam seu estado ao longo do tempo e agem segundo uma natureza aleatória. Diante da existência 
destas características, a simulação computacional torna uma alternativa adequada de modelagem, na medida em que esta consegue capturar com mais fidelidade o comportamento do sistema real (MEDINA e CHWIF, 2007).

A simulação computacional pode ser classificada em três categorias básicas: simulação de Monte Carlo, Contínua e de Eventos Discretos. A simulação de Monte Carlo utiliza geradores de números aleatórios para simular, não considerando explicitamente o tempo como uma variável. Entretanto, a simulação de eventos discretos e a simulação de eventos contínuos levam em consideração a mudança de estado do sistema ao longo do tempo (MEDINA e CHWIF, 2007).

De uma maneira geral, a simulação computacional apresenta diversas vantagens para a análise dos processos pelos seus gestores. São exemplos:

- Em muitos casos, o desenvolvimento de um modelo analítico matemático para analisar o processo é extremamente difícil de ser operacionalizado, tornando a simulação uma melhor alternativa de modelagem para este processo;

- Em muitos casos, o processo apresenta um comportamento dinâmico no tempo, modificando seu estado durante sua operação, tornando necessária incorporação da variabilidade no modelo que irá analisar o mesmo. Nesta situação, a simulação possibilita a incorporação satisfatória desta variabilidade no modelo;

- Simulações fornecem um ambiente seguro para se cometer erros, consequentemente aprender com os mesmos;

- Simulações possibilitam a experimentação e a verificação de diferentes cenários para o processo em análise;

- Pelas suas características de maior semelhança com o modelo real, a simulação permite maior aprendizagem por parte dos gestores (MAHBOUBIANA, 2010; LAGUNA e MARKLUND, 2004).

Existe uma grande quantidade de softwares que podem ser utilizados na simulação de processos. Uma lista de softwares livres de simulação pode ser encontrada no endereço eletrônico http://www.topology.org/soft/sim.html (HILL, 2008).

Apesar de ser fortemente ignorada por especialistas em modelagem, a planilha eletrônica fornece um ambiente diversificado e robusto para a maioria das necessidades de modelagem. Esta provê um meio para armazenar e gerenciar dados, executar análises estatísticas, conduzir importação e exportação de tabelas e gráficos, além de executar a interface com outros softwares mais poderosos (HILL, 2008).

Levando-se em consideração o custo de aquisição e a facilidade de aprendizagem, optou-se pela utilização do pacote SimQuick (http://www.prenhall.com/hartvigsen ). Este é uma aplicação do software Excel e permite a realização de simulação de processos dentro do ambiente de planilha 
Excel. SimQuick não é um suplemento do Excel (como um .xla), é um modelo de planilha no qual um usuário especifica os elementos de um modelo de simulação, a parametrização desses elementos, e as conexões entre os mesmos. Seu funcionamento pode ser compreendido em poucas horas de aulas. Pode ser descrito como um pacote de primeira geração. Portanto, apresenta varias limitações se comparado com softwares maduros e dedicados como o Extend, ARENA, ProModel. No entanto, mesmo diante das suas limitações, o SimQuick é bastante adequado como ferramenta para entender o funcionamento e as características dos processos. Pode ser visto como uma ferramenta inicial de simulação para análise de processos. Possibilita a estimação da capacidade, o tempo de espera, o tamanho da fila e o grau de utilização dos recursos, além de possibilitar a realização de análises estatísticas destas variáveis. Este software é utilizado em vários cursos de MBA nos Estados Unidos (LEV, 2002; HILL, 2008; HARTVIGSEN, 2005).

No SimQuick, a configuração do modelo de simulação é feita mediante o uso de alguns blocos de construção. O programa trabalha com cinco tipos de blocos. São eles:

- Bloco "Buffers": Funciona como uma entidade de estocagem no processo;

- Bloco "Entrances": Entidade para definir o processo de chegada de produtos ou clientes;

- Bloco “WorkStations": Esta entidade funciona como uma estação de trabalho que processa os produtos ou as pessoas em um processo;

- Bloco "Decision Points": Esta entidade é utilizada quando o objeto a ser processado pode seguir em mais de uma direção no processo;

- Bloco "Exits": Esta é utilizada quando o objeto já processado deixa o processo segundo um escala de tempo ou segundo uma distribuição estatística (HARTVIGSEN, 2005).

O preenchimento dos blocos de construção existentes no SimQuick se dá mediante o acionamento dos "botões de links" existentes no Painel de Controle, veja um exemplo deste painel na Figura 01. Cada "botão de link" conduz a uma planilha específica que deverá ser preenchida com os parâmetros pertinente ao modelo e bloco de construção (HARTVIGSEN, 2005). 


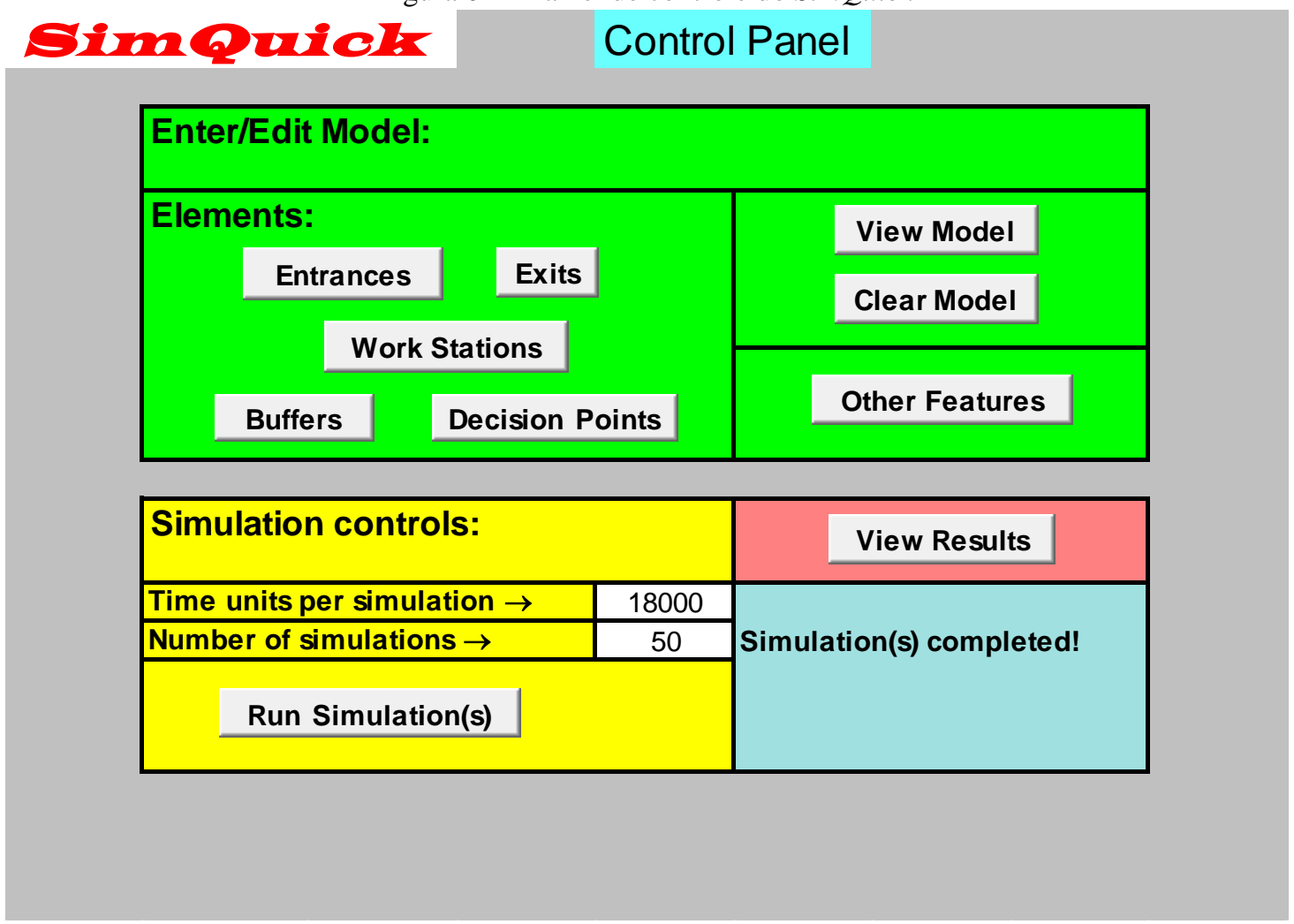

Fonte: Planilha do SimQuick

\subsection{Otimização por meio do planejamento e análise de experimentos}

Muitas das vezes, os analistas de um processo estão interessados em saber quais variáveis são importantes e em qual configuração delas resultará num menor custo. Varias perguntas deste tipo podem ser respondidas com a estatística experimental. O uso do Planejamento e Analise de Experimentos (Design of Experiments - DOE) é uma destas técnicas da estatística experimental que atualmente vem sendo usada em grande escala com o objetivo de melhorar o desempenho dos processos. O DOE utiliza várias ferramentas. Baseando-se em diversos autores da área de Estatística Industrial, os autores Galdamez e Carpinetti (2004) reportam as principais ferramentas de DOE e estas são apresentadas no Quadro 1. 
Quadro 1 - Principais ferramentas de DOE

\begin{tabular}{|c|c|}
\hline Ferramentas & Características \\
\hline Planejamento fatorial & Utilizada quando todas as combinações dos níveis dos fatores de controle são \\
realizadas.
\end{tabular}

Fonte: Quadro de Galdamez e Carpinetti (2004)

Considerando os objetivos propostos pelo trabalho e grau de utilização destas ferramentas em experimentos industriais, os autores discutem apenas os experimentos fatoriais neste trabalho.

Os planejamentos fatoriais podem ser analisados por meio de Modelos Lineares Gerais (General Linear Models). Nesta direção, os autores Mathews (2005), Barrentine (1999) e Mendenhall e Sincich (2012) apresentam esta abordagem quando explicam experimentos deste tipo. O uso da regressão pode ser visto como uma alternativa didática para o ensino de DOE, uma vez que a regressão trabalha com o conceito de variável dependente (correspondente à variável resposta para o DOE) e as variáveis independentes (correspondentes aos fatores controlados). Além disso, as relações entre possíveis causas e o efeito tornam-se mais claras nos modelos de regressão. Soma a estes aspectos, o fato de que, normalmente, os estudantes da graduação e profissionais da indústria já estão familiarizados com esta ferramenta estatística.

A seleção de um modelo de regressão deve ser feita de maneira a selecionar as variáveis que sejam significativas para o modelo. Para tanto, pode-se utilizar a analise variância que indicará quais fatores e quais interações entre eles são significativas (VIEIRA,2006).

A partir do conhecimento de quais variáveis e quais interações entre elas são significativas, pode-se utilizar Modelos Lineares Gerais para a estimação do efeito desejado.

Com objetivo de esclarecer a notação utilizada neste trabalho. Um experimento fatorial $2^{\mathrm{k}}$ com três fatores é apresentado. Nesta situação, tem-se o seguinte modelo completo:

- Fatores: $X_{1} ; X_{2} ; X_{3}$ 
- Termos de interação de segunda ordem:

- Termos de interação de terceira ordem $X_{123}$ (Interação entre os fatores 1,2 e 3 )

- Equação de regressão:

$$
\begin{aligned}
& \left.E(y)=\beta_{0}+\beta_{1} X_{1}+\beta_{2} X_{2}+\beta_{3} X_{3}+\beta_{4} X_{12}+\beta_{5} X_{13}+\beta_{6} X_{23}+\beta_{7} X_{123}\right) \\
& \text { Termos dos efeitos principais Termos dos efeitos de interação Termo do efeito de interação } \\
& \text { de segunda ordem de terceira ordem }
\end{aligned}
$$

Critérios utilizados na codificação dos níveis:

$$
\begin{array}{ll}
x & \left\{\begin{array}{l}
1 \text { para o primeiro nível } \\
-1 \text { para o segundo nível }
\end{array}\right. \\
X_{2} \quad\left\{\begin{array}{l}
1 \text { para o primeiro nível } \\
-1 \text { para o segundo nível }
\end{array}\right. \\
X_{3} \quad\left\{\begin{array}{l}
1 \text { para o primeiro nível } \\
\text {-1para o segundo nível }
\end{array}\right.
\end{array}
$$

Considerando os critérios expostos e a existência duas replicações este modelo completo pode ser descrito numa planilha eletrônica como demonstrado na Tabela 1.

Tabela 1 - Planilha contendo os dados de um experimento fatorial 23completo com 2 réplicas

\begin{tabular}{|c|c|c|c|c|c|c|c|c|}
\hline Resposta & Tratamento & X1 & X2 & X3 & X12 & X13 & X23 & X123 \\
\hline Valor 1 & 1 & 1 & 1 & 1 & 1 & 1 & 1 & 1 \\
\hline Valor 2 & 2 & 1 & 1 & -1 & 1 & -1 & -1 & -1 \\
\hline Valor 3 & 3 & 1 & -1 & 1 & -1 & 1 & -1 & -1 \\
\hline Valor 4 & 4 & 1 & -1 & -1 & -1 & -1 & 1 & 1 \\
\hline Valor 5 & 5 & -1 & 1 & 1 & -1 & -1 & 1 & -1 \\
\hline Valor 6 & 6 & -1 & 1 & -1 & -1 & 1 & -1 & 1 \\
\hline Valor 7 & 7 & -1 & -1 & 1 & 1 & -1 & -1 & 1 \\
\hline Valor 8 & 8 & -1 & -1 & -1 & 1 & 1 & 1 & -1 \\
\hline Valor 9 & 1 & 1 & 1 & 1 & 1 & 1 & 1 & 1 \\
\hline Valor 10 & 2 & 1 & 1 & -1 & 1 & -1 & -1 & -1 \\
\hline Valor 11 & 3 & 1 & -1 & 1 & -1 & 1 & -1 & -1 \\
\hline Valor 12 & 4 & 1 & -1 & -1 & -1 & -1 & 1 & 1 \\
\hline Valor 13 & 5 & -1 & 1 & 1 & -1 & -1 & 1 & -1 \\
\hline Valor 14 & 6 & -1 & 1 & -1 & -1 & 1 & -1 & 1 \\
\hline Valor 15 & 7 & -1 & -1 & 1 & 1 & -1 & -1 & 1 \\
\hline Valor 16 & 8 & -1 & -1 & -1 & 1 & 1 & 1 & -1 \\
\hline
\end{tabular}

Fonte: Pesquisa dos autores (2012) 
Os termos de interação existentes na planilha mostrada na Tabela 1 são obtidos pela multiplicação das respectivas variáveis $X_{1}, X_{2}$ e $X_{3}$. Por exemplo, o termo de interação $X_{12}$ é obtido pela multiplicação dos termos existentes na coluna de $X_{1}$ pelos termos da coluna de $X_{2}$.

A construção do modelo de regressão pode ser feita utilizando a ferramenta análise de dados disponível no programa Excel.

\section{Estratégia metodológica utilizada}

Este texto visa demonstrar uma estratégia de ensino desenvolvida pelos autores e apoiada nos trabalhos de Laguna \& Marklund (2004), Hartvigsen (2001) e Mendenhall\&Sincich (2012) para a análise de processos apoiada nas técnicas de simulação computacional de eventos discretos e no Planejamento e Análise de Experimentos. Esta estratégia foi aprimorada pela experiência docente de um dos autores que a utilizou parte desta estratégia em uma disciplina optativa dedicada a análise de processos, durante os últimos três anos. Por ter como principal finalidade a demonstração de uma dada estratégia de ensino, este trabalho é do tipo exploratório e de natureza descritiva.

Nesta estratégia, a análise de processos foi feita segundo um conjunto de atividades. Estas atividades podem ser agrupadas em quatro etapas. São elas:

- Primeira etapa - Identificação de oportunidades e definição do escopo do trabalho: por meio de entrevistas aos seus gestores, o grupo de alunos deverá escolher uma organização identificando oportunidades de melhoria na mesma. Para tanto, eles deverão reconhecer quais os principais processos existentes na organização investigada, selecionando alguns. Deverão ser mapeados para cada um desses processos os principais recursos, produtos e clientes. Como cada um desses processos normalmente está envolvido na geração de valor para os clientes externos ou internos, eles deverão realizar uma série de perguntas visando à identificação de oportunidades de melhoria sob esta perspectiva. Além disso, a escolha do processo que será analisado deverá considerar a facilidade de acesso aos dados e informações. Uma vez selecionado o processo, os limites dessa analise devem ser claramente estabelecidos.

- Segunda etapa - Documentação do Processo: uma vez que o escopo do processo a ser analisado foi estabelecido, o grupo deverá realizar o mapeamento detalhado do mesmo utilizando alguma ferramenta de diagramação. Dados referentes ao tempo de processamento de cada atividade devem ser levantados e estimados. De posse destas informações, realiza-se uma estimativa do tempo total de processamento, do fluxo de produção e da capacidade de processamento. Estas informações serão posteriormente confrontadas com as estatísticas obtidas pela simulação computacional como um instrumento para a verificação do modelo. 
- Terceira etapa - Avaliação do Processo por meio da Simulação Computacional: uma vez que o processo foi diagramado e o tempo de cada atividade foi estimado, pode-se proceder com a construção do modelo no software de simulação.

- Quarta etapa - Proposta de Redesenho: com o modelo do processo atual já configurado e verificado no software de simulação, pode-se proceder com a análise do processo propriamente dito. Esta pode ser feita modificando a configuração do processo e testando o impacto destas mudanças no tempo de processamento, no tempo de espera, no grau de utilização dos recursos e na capacidade de processamento por meio do DOE.

O Quadro 1 apresenta uma síntese das etapas e dos principais conceitos e ferramentas que devem ser utilizados em cada uma delas.

Quadro 1 - Síntese das etapas e dos principais conceitos e ferramentas utilizados

\begin{tabular}{|l|l|}
\hline Etapa & $\begin{array}{l}\text { Principais ferramentas e conceitos utilizados, e a referência } \\
\text { utilizada }\end{array}$ \\
\hline $\begin{array}{l}\text { Identificação de oportunidades e } \\
\text { definição do escopo do trabalho }\end{array}$ & Fluxograma \\
\hline Documentação do Processo & $\begin{array}{l}\text { Diagrama de rede; Mensuração do tempo e da capacidade } \\
\text { produtiva (LAGUNA e MARKLUND, 2004). }\end{array}$ \\
\hline $\begin{array}{l}\text { Avaliação do Processo por meio } \\
\text { da Simulação Computacional }\end{array}$ & $\begin{array}{l}\text { Estatística descritiva; distribuições de probabilidade; testes de } \\
\text { aderência; verificação de modelos de simulação (MEDINA e } \\
\text { CHWIF, 2007). } \\
\text { Software SimQuick (HARTVIGSEN, 2005). }\end{array}$ \\
\hline Proposta de Redesenho & $\begin{array}{l}\text { Perdas em Processos (LAGUNA e MARKLUND, 2004); } \\
\text { ANOVA(VIEIRA,2006); Planejamento e Analise de } \\
\text { Experimentos (MENDENHALL \& SINCICH, 2012) }\end{array}$ \\
\hline
\end{tabular}

\section{Estudo de Caso}

Com intuito de ilustrar a estratégia metodológica utilizada para análise de processos, esta seção descreve à aplicação das quatro etapas propostas em uma empresa logística. Esta intervenção foi feita por um grupo de alunos durante uma disciplina de análise de processos. A empresa analisada é um operador logístico que atua no transporte rodoviário de bens para a região sudeste do Brasil.

\subsection{Primeira Etapa - Identificação de oportunidades e definição do escopo do trabalho}

Para melhor conhecimento do ambiente de trabalho e de seus processos foi feita um visita à empresa. O acesso a informações foi facilitado pela entrevista realizada com o diretor da área de planejamento e pela presença constante de um dos alunos na empresa (contratado como estagiário).

Após esta entrevista e considerando sua importância para a organização estudada, o grupo escolheu o setor de expedição de documentos para a realização do estudo, uma vez que o mesmo é 
responsável pela documentação legal dos serviços prestados. Em vista disso, o segundo entrevistado foi um funcionário do setor de expedição que ajudou o grupo a ter um melhor conhecimento das atividades realizadas nesta área.

Basicamente a função do setor de expedição é emitir e organizar os documentos fiscais necessários para a prestação de serviços de transporte. Isso inclui: emissão do Conhecimento de Transporte Rodoviário de Carga (CTRC), emissão do Recibo de Pagamento para Autônomo (RPA), emissão do Manifesto de Carga (Manifesto), emissão da Solicitação de Monitoramento Preventivo (SMP) e organização dos documentos impressos na ordem exigida pela lei (incluindo as notas fiscais dos produtos).

A análise da entrevista realizada com o gestor desta organização apontou um questionamento sobre a quantidade de funcionários e a carga horária de trabalho ideal para este setor. Assim, utilizando os dados registrados pelo cartão de ponto no período de 16 de fevereiro de 2012 a 15 de abril de 2012, realizou-se um levantamento histórico das entradas e saídas dos funcionários. Estes dados revelaram que em vários dias, alguns funcionários chegaram à empresa, em horários antecipados, gerando um custo adicional por hora extra. Existiram também casos de horas extras, após o horário normal de expediente.

Por se tratar de um trabalho noturno em que os documentos devem ser elaborados o mais rapidamente possível para a expedição das mercadorias que chegaram durante o dia, em alguns dias, os funcionários saíram mais cedo do expediente, uma vez que todas as tarefas daquele dia foram concluídas antes do término do horário normal. Como não há um desconto no salário do funcionário, este evento acaba gerando um custo desnecessário para a organização. Há ainda um gasto com pagamento de adicional noturno, que ocorre devido aos funcionários trabalharem após as $22 \mathrm{~h}$.

Diante destes fatos, o grupo entendeu que o problema a ser estudado no processo consiste na redução de custos com os funcionários existentes nos processos de expedição realizados pela equipe do turno noturno.

\subsection{Segunda Etapa - Documentação do Processo}

Após a definição do escopo de trabalho, foi realizado um mapeamento dos processos. Uma vez que o escopo do processo foi definido, realizou-se um mapeamento detalhado do mesmo. O setor de expedição conta com seis funcionários dentre os quais um trabalha no turno diurno (9h às 15h) e os outros cinco no turno noturno. Dos que trabalham no período da noite, tem-se um coordenador e quatro auxiliares de expedição. O coordenador e um dos auxiliares chegam às $17 \mathrm{~h} e$ os outros três auxiliares chegam às $18 \mathrm{~h}$. Todos trabalham de segunda a sexta-feira, seis horas por dia, e realizam revezamento aos sábados e domingos. O coordenador é responsável principalmente 
pela emissão do SPM, RPA e Manifesto. Dois auxiliares fazem a emissão do CTRC e Manifestos de Cargas e os outros dois são responsáveis pela conferência e organização dos documentos para viagem. Por escolha dos integrantes do setor, há uma rotatividade nas tarefas realizadas pelos auxiliares.

O fluxograma do processo de expedição é mostrado na Figura 2 deste trabalho.

Figura 2 - Fluxograma do processo de expedição de notas fiscais

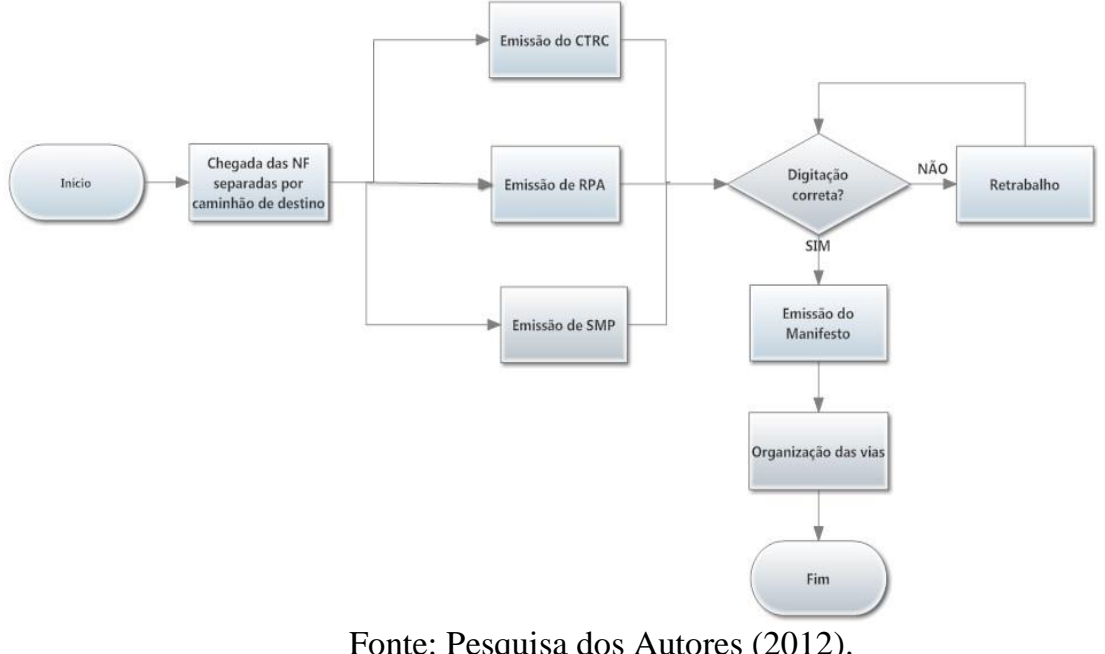

Fonte: Pesquisa dos Autores (2012).

Com o objetivo de compreender melhor o processo apresentado na Figura 3, uma análise do tempo gasto por cada tipo de atividade foi feita. Para tanto, um diagrama de PERT foi elaborado e o mesmo é apresentado na Figura 3 e na Tabela 2. Este diagrama baseou-se em tempos médios.

Figura 03 - Diagrama de PERT do processo de expedição de documentos

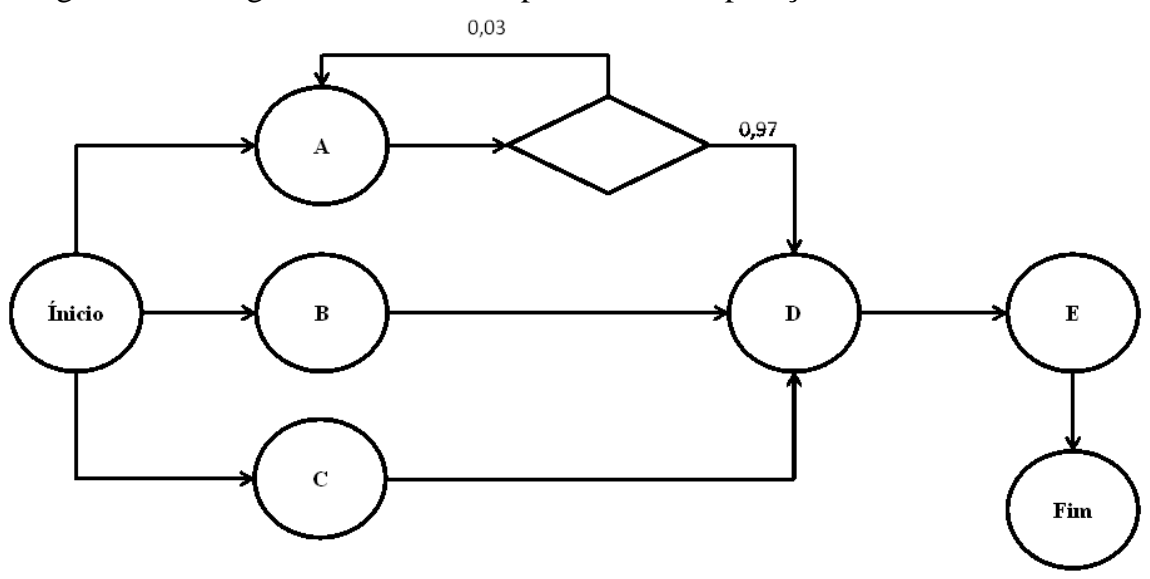

Fonte: Pesquisa dos Autores (2012). 
Tabela 2 - Descrição das atividades mostradas no Diagrama PERT

\begin{tabular}{|c|l|l|l|c|}
\hline Atividade & Descrição da Atividade & Recursos & $\begin{array}{l}\text { Tempo de } \\
\text { Execução (min) }\end{array}$ & $\begin{array}{l}\text { Antecessoras } \\
\text { Imediatas }\end{array}$ \\
\hline A & Emissão de CTRC & Auxiliar & $02: 12$ & - \\
\hline B & Emissão de RPA & Coordenador & $00: 15$ & - \\
\hline C & Emissão de SMP & Coordenador & $02: 06$ & - \\
\hline D & Emissão de Manifesto & Auxiliar/Coordenador & $01: 05$ & A,B,C \\
\hline E & Organização das Vias & Auxiliar & $01: 00$ & D \\
\hline
\end{tabular}

Fonte: Pesquisa dos Autores (2012).

Baseando-se no diagrama de PERT apresentado na Figura 4, na Tabela 2 e na demanda média diária para cada tipo de documento gerado neste processo (CTRC, RPA, SMP e Manifesto de Carga), o tempo médio diário gasto por cada funcionário na realização das atividades foi calculado. Estes dados são apresentados na Tabela 3.

Tabela 3 - Cálculo do tempo teórico gasto diário por funcionário em cada documento.

\begin{tabular}{|l|l|c|c|c|c|}
\hline Documento & \multicolumn{2}{|c|}{$\begin{array}{c}\text { Recurso } \\
\text { Principal (Cp) }\end{array}$} & $\begin{array}{c}\text { Carga de } \\
\text { Trabalho Tp } \\
\text { (minutos por item) }\end{array}$ & $\begin{array}{c}\text { Demanda diária } \\
\text { (unidades) }\end{array}$ & $\begin{array}{c}\text { Tempo Teórico } \\
\text { Gasto por } \\
\text { Funcionário } \\
\text { (minutos) }\end{array}$ \\
\hline CTRC & Auxiliar & 2 & $2,200 \times 1,03$ & 290 & 328,57 \\
\hline RPA & Coordenador & 1 & 0,250 & mínimo de 0 máximo de 15 & 0 \\
\hline SMP & Coordenador & 1 & 2,100 & mínimo de 0 máximo de 15 & 0 \\
\hline $\begin{array}{l}\text { Manifesto } \\
\text { de Carga }\end{array}$ & $\begin{array}{l}\text { Auxiliar/ } \\
\text { Coordenador }\end{array}$ & 5 & 1,083 & 15 & 31,5 \\
\hline $\begin{array}{l}\text { Via } \\
\text { Organizada }\end{array}$ & Auxiliar & 2 & 1,000 & 15 & 7,2 \\
\hline
\end{tabular}

Fonte: Pesquisa dos Autores (2012).

Com relação aos dados coletados, faz-se necessário a apresentação de algumas observações e esclarecimentos:

- Para este estudo foram considerados os dados dos auxiliares que realizaram digitação do conhecimento analítico em grandes escalas, dessa forma o coordenador do setor não foi analisado. O quarto auxiliar não foi considerado para a média, pois em parte do período analisado estava de férias e no tempo restante não foi digitada uma quantidade significativa de CTRCs;

- No período analisado a empresa emitiu em média 15 Manifestos de carga por dia. Observou-se que um operador demora em média 1 minuto e 5 segundos para a operação de um manifesto (média feita a partir de 12 emissões de um funcionário). De acordo com a Tabela 01 deste trabalho, o tempo teórico gasto por funcionário na digitação de Manifesto de Carga é de 3,2 minutos, o que, se comparado ao tempo total de trabalho, mostra-se irrelevante; 
- O tempo de emissão de RPA e SMP também é irrelevante já que, salvo exceções, estes documentos são emitidos pelo coordenador. Além disso, estão em menor quantidade que os Manifestos e levam um tempo médio de 15 segundos e 02 minutos e 06 segundos respectivamente (médias calculadas a partir de 4 e 11 emissões respectivamente). Como pode ser visto na Tabela 01 deste trabalho, o tempo teórico gasto por funcionário para estas atividades é relativamente pequeno se comparado ao tempo de digitação de CTRCs;

- Sábados e domingos foram desconsiderados devido ao número reduzido de funcionários e tarefas a serem realizadas.

\subsection{Terceira Etapa - Avaliação do Processo por meio da Simulação Computacional}

Uma vez que a análise será feita no processamento das CTRCs, buscou-se definir um modelo conceitual do processo que retratasse as atividades do processo atual segundo a lógica utilizada pelo programa SimQuick. Posteriormente, procedeu-se com a definição dos dados de entrada do sistema. Para tanto, realizou-se uma análise estatística do tempo de processamento dos operadores. Esta análise revelou dois grupos distintos de operadores: no primeiro grupo encontramse os operadores experientes com um grau de destreza superior ao segundo grupo e por isso apresentam uma taxa de processamento expressa por uma distribuição normal com média de 103 segundos e desvio padrão de 19 segundos por CTRC e uma taxa percentual de retrabalho de $2 \%$. O segundo grupo é constituído por operadores com uma experiência menor nesta atividade, portanto realizam as atividades de emissão de CTRCs gastando um tempo maior, com uma taxa de processamento expressa por uma distribuição normal com média de 141 segundos e desvio padrão de 31 segundos por CTRC e percentual de retrabalho em aproximadamente $4 \%$ dos documentos. Como a média diária de CTRCs enviadas para o setor é da ordem de 290, e os funcionários não compartilham do mesmo pacote de notas, cada operador inicia a simulação com 145 unidades. Nesta configuração considerou-se que todas as notas fiscais já estão disponíveis no inicio do período de trabalho. A Figura 04 e a Tabela 04 retratam o modelo conceitual para modelagem deste processo utilizando o SimQuick. 
Figura 4 - Modelo conceitual do processo atual utilizando o SimQuick.

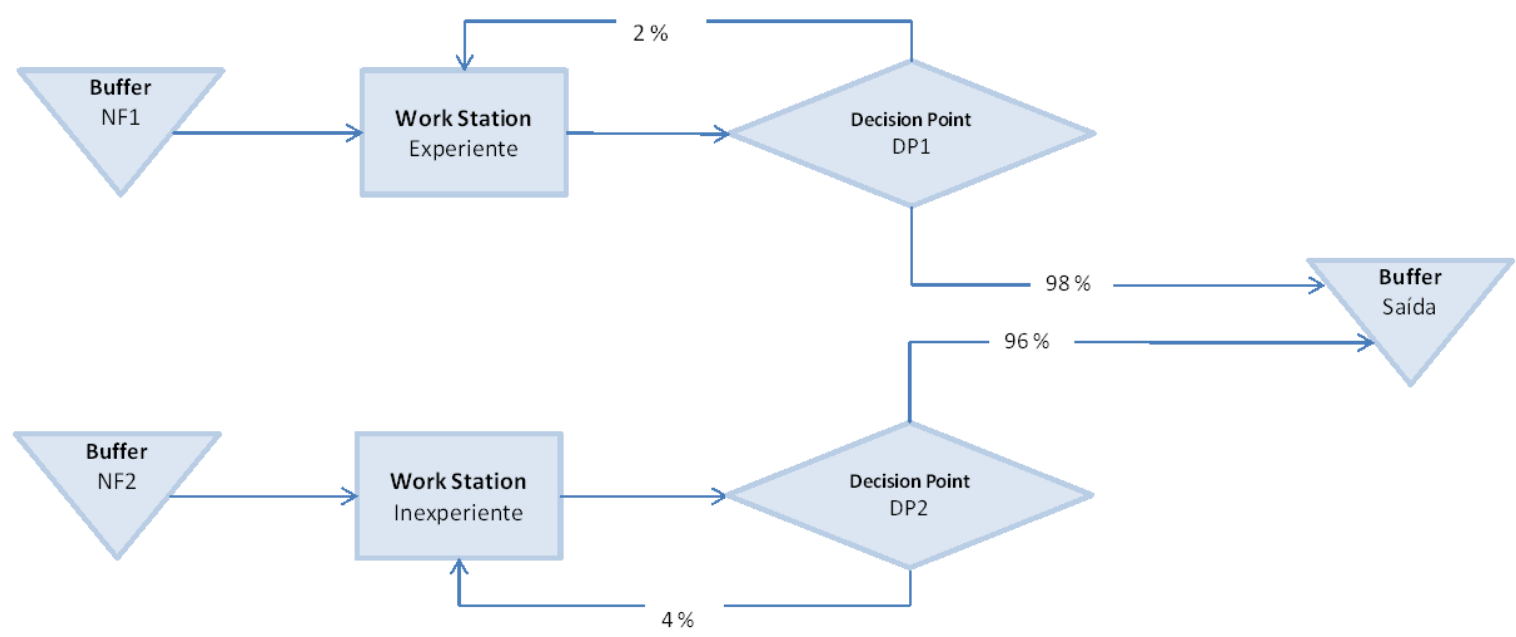

Fonte: Pesquisa dos Autores (2012).

Tabela 4 - Detalhamento do modelo conceitual do processo atual utilizando o SimQuick

\begin{tabular}{|c|c|c|}
\hline $\begin{array}{l}\text { Bloco de construção } \\
\text { utilizado }\end{array}$ & Nome & Características \\
\hline \multirow{3}{*}{ Buffers } & $N F 1$ & Valor inicial de 145 unidades \\
\hline & $N F 2$ & Valor inicial de 145 unidades \\
\hline & Saida & Capacidade ilimitada \\
\hline \multirow{2}{*}{ WorkStations } & Experiente & $\begin{array}{l}\text { Tempo de Processamento segue umadistribuição normal commédia de } 103 \\
\text { segundos e desvio padrão de } 19 \text { segundos por CTRC }\end{array}$ \\
\hline & Inexperiente & $\begin{array}{l}\text { Tempo de Processamento segue umadistribuição normal com média de } 141 \\
\text { segundos e desvio padrão de } 31 \text { segundos por CTRC }\end{array}$ \\
\hline \multirow{2}{*}{ Decision Points } & $D P 1$ & Retrabalho de $2 \%$ \\
\hline & $D P 2$ & Retrabalho de $4 \%$ \\
\hline
\end{tabular}

Fonte: Pesquisa dos Autores (2012).

Após a definição do modelo conceitual do processo e dos parâmetros de entrada, procedeuse com a simulação propriamente dita. Alguns resultados obtidos com a mesma são apresentados seguir.

Tabela 5 - Estatística Descritiva das horas diárias trabalhadas por operador

\begin{tabular}{|c|c|c|c|c|c|c|c|}
\hline $\begin{array}{c}\text { Tipo de } \\
\text { Operador }\end{array}$ & Mínimo & $1^{o}$ Quartil & Média & Mediana & $3^{\circ}$ Quartil & Máximo & $\begin{array}{l}\text { Desvio } \\
\text { Padrão }\end{array}$ \\
\hline Experiente & 3,98 & 4,19 & 4,23 & 4,23 & 4,29 & 4,52 & 0,087 \\
\hline Inexperiente & 5,53 & 5,83 & 5,89 & 5,91 & 6,00 & 6,00 & 0,112 \\
\hline
\end{tabular}

Observa-se pela Tabela 5 que o trabalhador mais experiente necessita de um tempo menor do que às seis horas disponíveis para processar as 145 CTRCs. Por outro lado, o operador inexperiente, muitas das vezes, gasta quase todo o tempo disponível para processar as 145 CTRCs. 
Tabela6 - Estatística Descritiva do percentual retrabalhado por operador

\begin{tabular}{|cccccccc|}
\hline Fatores & Mínimo & $\mathbf{1}^{\boldsymbol{o}}$ Quartil & Média & Mediana & $\mathbf{3}^{\boldsymbol{o}}$ Quartil & Máximo & $\begin{array}{c}\text { Desvio } \\
\text { Padrão }\end{array}$ \\
\hline Experiente & $0,0 \%$ & $1,4 \%$ & $2,2 \%$ & $2,1 \%$ & $3,3 \%$ & $6,9 \%$ & 0,0137 \\
Inexperiente & $0,7 \%$ & $2,8 \%$ & $3,8 \%$ & $3,4 \%$ & $4,8 \%$ & $6,9 \%$ & 0,0155 \\
\hline
\end{tabular}

A Tabela 6 revela a variação do percentual retrabalhado por cada operador. Apesar do percentual médio de retrabalho ser maior para o funcionário pouco experiente, os dois funcionários apresentam uma variação grande.

A partir destes dados, os custos com mão-de-obra deste processo ${ }^{1}$ foram estimados e são apresentados na Tabela 7.

Tabela 7 - Custos com mão-de-obra do processo atual.

\begin{tabular}{|l|r|r|}
\hline \multicolumn{1}{|c|}{ Descrição } & \multicolumn{3}{|}{$\begin{array}{r}\text { Situação Atual: 1 funcionário pouco experiente e 1 funcionário experiente } \\
\text { trabalhando 6horas por dia e iniciando o expediente às 18h. }\end{array}$} \\
\hline Tipo de gasto & $1^{\circ}$ Funcionário (Pouco Experiente) & $2^{\circ}$ Funcionário (Experiente) \\
\hline Hora normal trabalhada & $\mathrm{R} \$ 580,00$ & $\mathrm{R} \$ 420,00$ \\
\hline Hora extra trabalhada & $\mathrm{R} \$ 0,00$ & $\mathrm{R} \$ 0,00$ \\
\hline Adicional noturno & $\mathrm{R} \$ 36,00$ & $\mathrm{R} \$ 4,00$ \\
\hline Abono & $\mathrm{R} \$ 20,00$ & $\mathrm{R} \$ 180,00$ \\
\hline Total por funcionário & $\mathrm{R} \$ 636,00$ & $\mathrm{R} \$ 604,00$ \\
\hline Total gasto & $\mathrm{R} \$ 1.240,00$ \\
\hline
\end{tabular}

Fonte: Pesquisa dos Autores (2012).

\subsection{Quarta Etapa - Proposta de Redesenho}

Uma vez que o processo já foi modelado usando o SimQuick, busca-se uma melhor compreensão dos fatores que afetam os custos com a mão de obra. Busca-se também definir, por meio do DOE, quais mudanças seriam necessárias para se obter o menor custo. Para tanto, torna-se necessário identificar quais fatores que podem impactar positivamente na redução dos custos. Nesta direção, três fatores que poderiam ser trabalhados pela simulação e que podem afetar significativamente os custos foram identificados, ou seja:

- O grau de experiência do operador influencia no tempo gasto no processamento dos documentos e na quantidade retrabalhada, consequentemente impacta no gasto com a mão-de-obra;

- A hora de inicio do turno de trabalho pode influenciar o gasto com adicional noturno e abonos despendidos, portanto também impacta no gasto com a mão-de-obra;

- A duração do turno também influencia o valor gasto com os funcionários.

\footnotetext{
${ }^{1}$ Por solicitação da empresa analisada, os custos mostrados não são os reais, uma vez que estão multiplicados por uma constante. Entretanto os mesmos obedecem à proporção real existente entre eles.
} 
Levando-se em consideração estes fatores procedeu-se com o planejamento do experimento fatorial. Para tanto, definiu-se os níveis que deverão ser trabalhados e o tipo de planejamento fatorial que será conduzido e o Quadro 1 retrata esta informação.

Quadro 1 - Detalhamento dos fatores utilizados

\begin{tabular}{|l|c|c|c|c|}
\hline Fator & Tipo de Variável & Primeiro Nível & Segundo Nível & Terceiro Nível \\
\hline $\begin{array}{l}\text { Hora de inicio do turno de } \\
\text { trabalho }\left(X_{1}\right)\end{array}$ & Categórica & $17 \mathrm{~h}$ & $18 \mathrm{~h}$ & \\
\hline Duração do turno $\left(X_{2}\right)$ & $\begin{array}{c}\text { Numérica } \\
\text { Contínua }\end{array}$ & $5 \mathrm{~h}$ & $6 \mathrm{~h}$ & \\
\hline $\begin{array}{l}\text { Tipos de Operadores }\left(X_{3} \mathrm{e}\right. \\
\left.X_{4}\right)\end{array}$ & Categórica & Ambos experientes & $\begin{array}{c}\text { Um experiente } \\
\text { e o outro } \\
\text { inexperiente }\end{array}$ & $\begin{array}{c}\text { Ambos } \\
\text { inexperientes }\end{array}$ \\
\hline
\end{tabular}

Fonte: Pesquisa dos Autores (2012).

Levando-se em consideração os fatores e os seus níveis, escolheu-se o experimento fatorial misto $3^{1} 2^{2}$ completo com duas replicações e apenas um bloco. A variável resposta utilizada foi o custo com a mão de obra. Definiu-se o Planejamento de Experimento com estes fatores e a Tabela 8 mostra os tratamentos realizados e os custos com a mão de obra (y) obtidos com as simulações.

Tabela 8 - Tratamentos realizados e os custos com a mão de obra(y) obtidos com as simulações.

\begin{tabular}{|c|c|c|c|c|c|c|}
\hline \multirow{2}{*}{$\begin{array}{l}\text { Ordem } \\
\text { padrão }\end{array}$} & \multirow{2}{*}{$\begin{array}{l}\text { № de } \\
\text { Blocos }\end{array}$} & \multirow{2}{*}{$\begin{array}{l}\text { Horário de } \\
\text { Início } \\
\left(X_{1}\right) \\
\end{array}$} & \multirow{2}{*}{$\begin{array}{c}\text { Duração do } \\
\text { turno } \\
\left(X_{2}\right) \\
\end{array}$} & \multirow{2}{*}{$\begin{array}{c}\text { Tipos de } \\
\text { Operadores } \\
\left(\mathrm{X}_{3} \text { e } \mathrm{X}_{4}\right)\end{array}$} & \multicolumn{2}{|c|}{$Y$ (reais) } \\
\hline & & & & & 1 & 2 \\
\hline 1 e 13 & 1 & $17 \mathrm{~h}$ & 5 & 2 inexp & $1.226,99$ & $1.223,26$ \\
\hline 2 e 14 & 1 & $17 \mathrm{~h}$ & 6 & 2 inexp & $1.234,51$ & $1.235,31$ \\
\hline 3 e 15 & 1 & $18 \mathrm{~h}$ & 5 & 2 inexp & $1.262,93$ & $1.262,78$ \\
\hline 4 e 16 & 1 & $18 \mathrm{~h}$ & 6 & 2 inexp & $1.275,83$ & $1.275,36$ \\
\hline 5 e 17 & 1 & $17 \mathrm{~h}$ & 5 & $2 \exp$ & $1.000,00$ & $1.000,00$ \\
\hline 6 e 18 & 1 & $17 \mathrm{~h}$ & 6 & $2 \exp$ & $1.200,00$ & $1.200,00$ \\
\hline 7 e 19 & 1 & $18 \mathrm{~h}$ & 5 & $2 \exp$ & $1.009,79$ & $1.009,60$ \\
\hline 8 e 20 & 1 & $18 \mathrm{~h}$ & 6 & $2 \exp$ & $1.210,66$ & $1.209,31$ \\
\hline 9 e 21 & 1 & $17 \mathrm{~h}$ & 5 & 1inexp 1exp & $1.111,84$ & $1.113,19$ \\
\hline 10 e 22 & 1 & $17 \mathrm{~h}$ & 6 & 1inexp 1exp & $1.217,88$ & $1.217,58$ \\
\hline 11 e 23 & 1 & $18 \mathrm{~h}$ & 5 & 1inexp 1exp & $1.134,21$ & $1.139,89$ \\
\hline 12 e 24 & 1 & $18 \mathrm{~h}$ & 6 & 1 inexp $1 \exp$ & $1.242,39$ & $1.242,84$ \\
\hline
\end{tabular}

Fonte: Pesquisa dos Autores (2012).

A análise de variância foi feita por meio de planilhas eletrônicas utilizando a abordagem proposta pela autora Vieira (2006). Os resultados obtidos pela análise de variância feita com os dados obtidos pelas simulações são mostrados na Tabela 9. 
Tabela 9 - Análise de variância feita com os dados obtidos pelas simulações.

\begin{tabular}{|c|c|c|c|c|c|}
\hline Fatores & $\begin{array}{l}\text { Graus de } \\
\text { Liberdade }\end{array}$ & $\begin{array}{l}\text { Soma de } \\
\text { quadrados }\end{array}$ & $\begin{array}{l}\text { Quadrado } \\
\text { médio }\end{array}$ & $F_{\text {parcial }}$ & $P_{\text {valor }}$ \\
\hline Tipos de Operadores* & 2 & 83.755 & 41.878 & $19.649,77$ & 0 \\
\hline Hora de inicio * & 1 & 3.626 & 3.626 & $1.701,54$ & 0 \\
\hline Duração do turno* & 1 & 66.908 & 66.908 & $31.394,46$ & 0 \\
\hline $\begin{array}{l}\text { Interação entre Tipos de Operadores e } \\
\text { Tipos de Operadores } *\end{array}$ & 2 & 862 & 431 & 202,31 & 0 \\
\hline $\begin{array}{l}\text { Interação entre Tipos de Operadores e } \\
\text { Duração do turno* }\end{array}$ & 2 & 35.676 & 17.838 & $8.369,89$ & 0 \\
\hline $\begin{array}{l}\text { Interação entre Hora de inicio e Duração do } \\
\text { turno }\end{array}$ & 1 & 2 & 2 & 1,01 & 0,334 \\
\hline $\begin{array}{l}\text { Interação entre Tipos de Operadores e } \\
\text { Duração do turno e Hora de inicio }\end{array}$ & 2 & 2 & 1 & 0,55 & 0,593 \\
\hline Erro & 12 & 26 & 2 & & \\
\hline Total & 23 & 190.857 & & & \\
\hline
\end{tabular}

* Fatores considerados significativos.

Fonte: Pesquisa dos Autores (2012).

Com os resultados mostrados na Tabela 8 é possível definir os fatores que são significativos e com estes resultados pode-se definir o modelo de regressão que definirá o custo com a mão de $\operatorname{obra}(y)$. Este é apresentado a seguir:

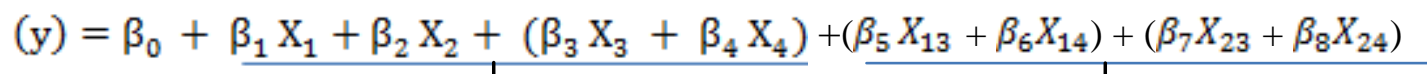

$$
\begin{aligned}
& \text { Termos de primeira ordem onde } X_{2} \text { refere-se ao fator horário de inicio; } Z_{z} \text { refere-se ao fator duração do turno; e os termos } Z_{\Sigma} \text { e } X_{4}
\end{aligned}
$$

\footnotetext{
Termos com interação de segunda ordem onde $X_{2 \Sigma}$ e $X_{24}$ significam a interação entre o fator $X_{12}$ e o fator tipo de operadores composto pelos termos $X_{z}$ e $X_{4} ; X_{z z}$ e $X_{z}$ significam a interação entre o fator $X_{z}$ e o fator tipo de operadores composto pelos termos $X_{\mathrm{x}} \mathrm{e} X_{4}$.
}

Critérios utilizados na codificação dos níveis para o preenchimento da planilha no Excel que utilizará a ferramenta análise de dados para o uso da técnica de regressão:

$$
\begin{gathered}
X_{1}\left\{\begin{array}{l}
-1 \quad \text { para o primeiro nível - Hora de inicio do turno às } 17 \mathrm{~h} \\
1 \text { para o segundo nível - Hora de inicio do turno às } 18 \mathrm{~h}
\end{array}\right. \\
X_{3} \text { e } X_{4}\left\{\begin{array}{l}
-1 \quad \text { para o primeiro nível - Duração do turnode } 5 \mathrm{~h} \\
1 \quad \text { para o segundo nível - Duração do turno de } 6 \mathrm{~h}
\end{array}\right. \\
\left\{\begin{array}{l}
\text { no primeiro nível - Ambos operadores experientes } X_{3}=1 \text { e } X_{4}=0 \\
\text { no segundo nível - Um experiente e o outro inexperiente } X_{3}=0 \text { e } X_{4}=0
\end{array}\right.
\end{gathered}
$$


Com o propósito de verificar a adequação do modelo de regressão utilizado, utilizou-se a ferramenta análise de dados disponível no Software Excel. Com esta ferramenta os autores verificaram se os resíduos são independentes, têm média zero, apresentam uma variância constante e estão normalmente distribuídos. Os resultados destas análises são apresentados a seguir.

Tabela 10 - Anova para o modelo de regressão selecionado

ANOVA

\begin{tabular}{|c|c|c|c|c|c|c|}
\hline & $g l$ & & $S Q$ & $M Q$ & $F$ & F de significação \\
\hline Regressão & & 8 & 190827.4218 & 23853.42773 & 11904.27151 & $4.25819 \mathrm{E}-27$ \\
\hline Resíduo & & 15 & 30.05655705 & 2.00377047 & & $\pi$ \\
\hline Total & & 23 & 190857.4784 & & & \\
\hline
\end{tabular}

O baixo nível de significância mostrado pela Anova na Tabela 10 aponta que o modelo de regressão é válido para prever o custo de mão de obra $(y)$.

O modelo de regressão proposto apresenta um $R^{2}=\frac{190827,42}{190857_{i} 47}$ de $99.98 \%$, indicando que aproximadamente $99.98 \%$ da variação existente no custo de mão de obra $(y)$ pode ser explicada pela equação de regressão.

O Gráfico 1 mostra o gráfico de probabilidade dos resíduos padronizados. Como a maioria dos pontos formam uma reta, pode-se inferir que os resíduos se seguem uma distribuição normal.

Gráfico1- Gráfico de probabilidade dos resíduos padronizados

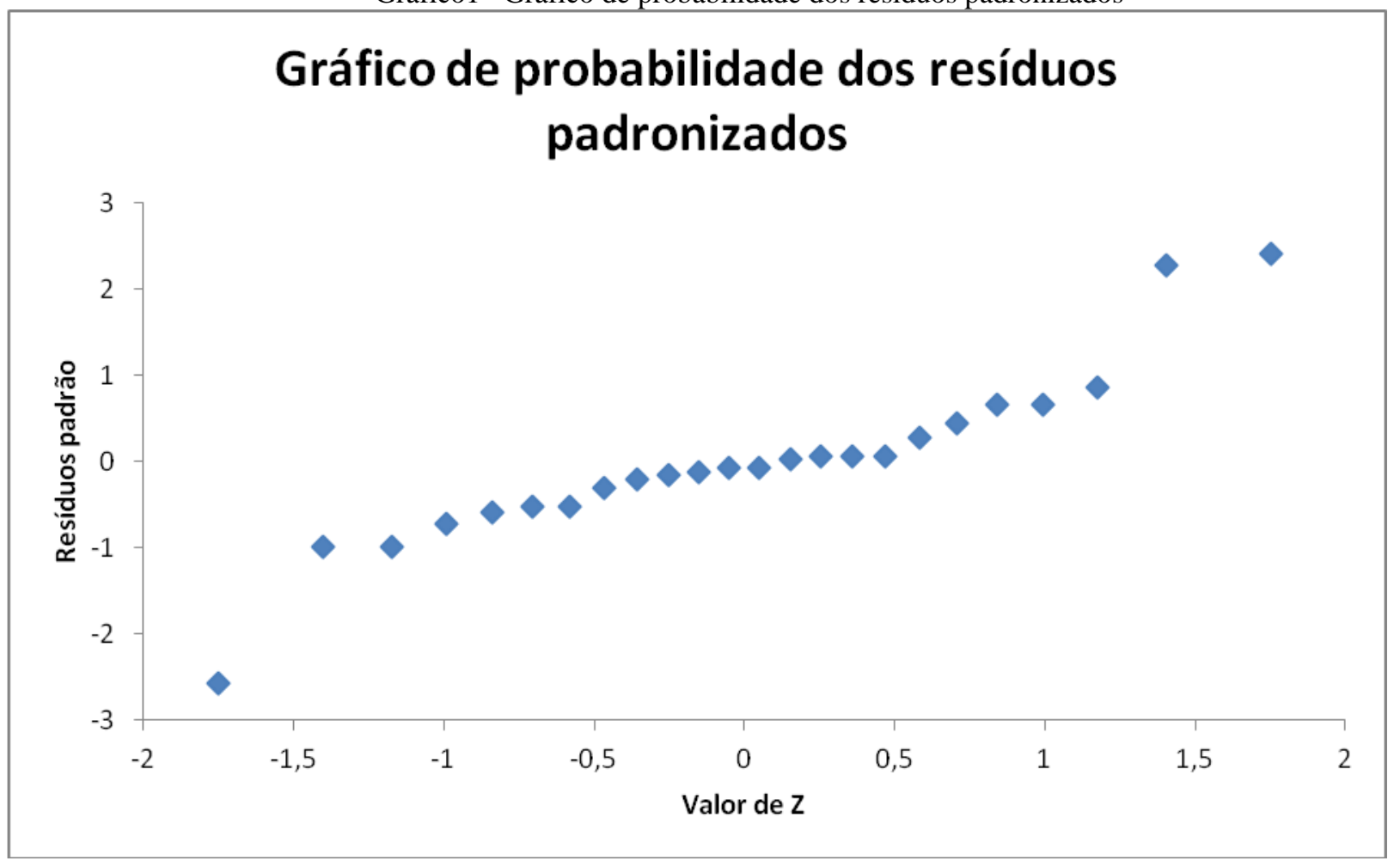

Fonte: Pesquisa dos Autores (2012). 
O Gráfico 2mostra o diagrama de dispersão dos resíduos padronizados e este indica que os resíduos apresentam uma variância constante.

Gráfico2 - Diagrama de dispersão por ordem de coleta dos resíduos padronizados

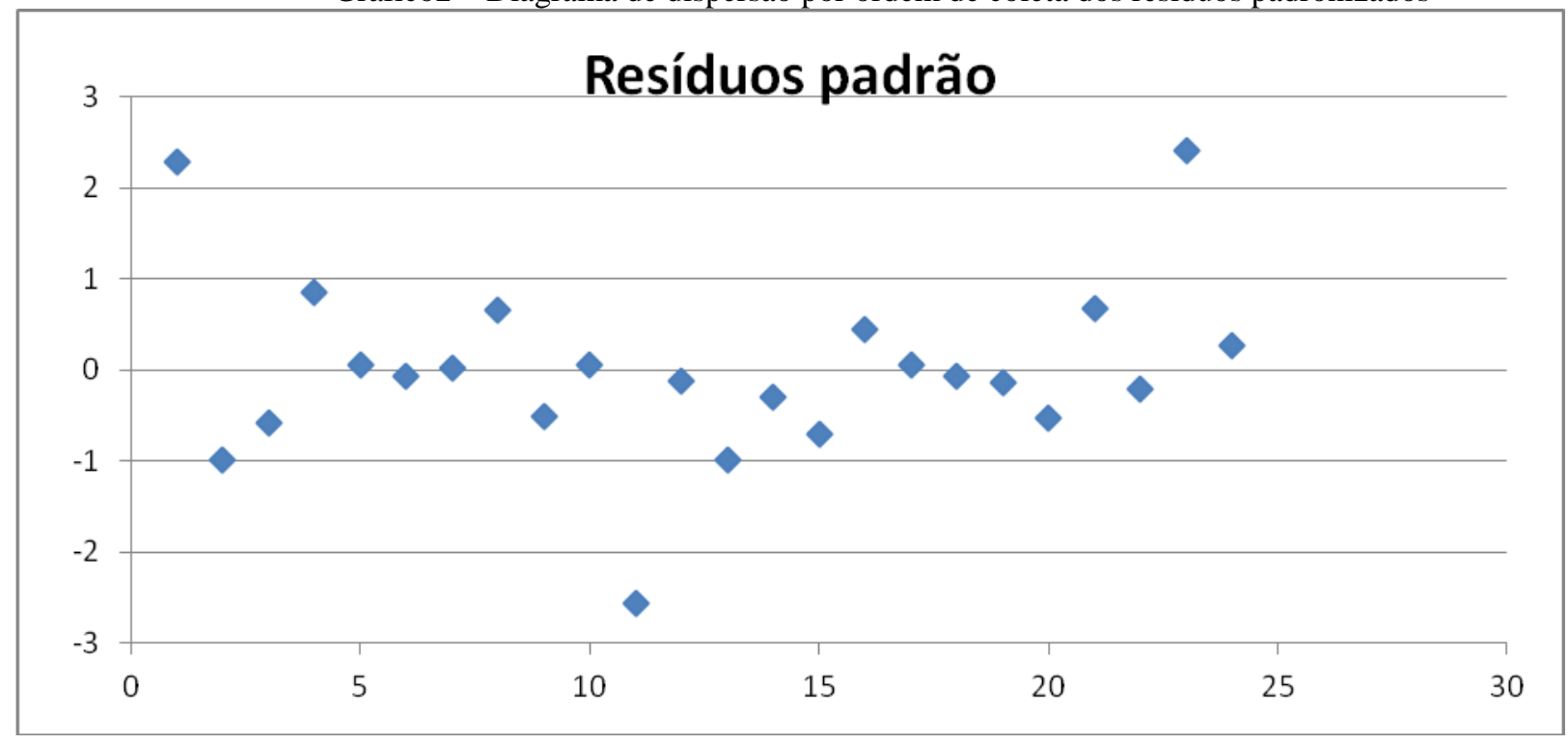

Fonte: Pesquisa dos Autores (2012).

Pelo exposto anteriormente, pode-se afirmar que o modelo de regressão se mostra adequado para os dados uma vez que os resíduos têm média zero, apresentam uma variância constante e estão normalmente distribuídos. Portanto o custo médio da mão de obra pode ser determinado pela equação de regressão descrita a seguir. De modo semelhante os coeficientes da equação de regressão foram obtidos pela ferramenta análise de dados disponível no Software Excel:

$(y)=1.177,5+12,4 X_{1}+52,7 X_{2}+72,1 X_{3}-72,6 X_{4}+7.2 X_{13}-7.4 X_{14}-47.1 X_{23}+47.4 X_{24}$

Tabela 11 - Coeficientes da equação de regressão.

\begin{tabular}{lcccrrr}
\hline & Coeficientes & $\begin{array}{c}\text { Erro } \\
\text { padrão }\end{array}$ & Stat t & valor-P & \multicolumn{1}{c}{$95 \%$} & \multicolumn{1}{c}{$95 \%$} \\
inferiores & superiores \\
\hline$\beta_{0}$ & $1.177,5$ & 0,5005 & $2.352,7$ & 0,000 & 1176,41 & 1178,54 \\
$\beta_{1}$ & 12,4 & 0,5005 & 24,7 & 0,000 & 11,29 & 13,42 \\
$\beta_{2}$ & 52,7 & 0,5005 & 105,3 & 0,000 & 51,63 & 53,76 \\
$\beta_{3}$ & 72,1 & 0,7078 & 101,9 & 0,000 & 70,64 & 73,65 \\
$\beta_{4}$ & $-72,6$ & 0,7078 & $-102,5$ & 0,000 & $-74,07$ & $-71,05$ \\
$\beta_{5}$ & 7,2 & 0,7078 & 10,2 & 0,000 & 5,74 & 8,76 \\
$\beta_{6}$ & $-7,4$ & 0,7078 & $-10,5$ & 0,000 & $-8,94$ & $-5,93$ \\
$\beta_{7}$ & $-47,1$ & 0,7078 & $-66,5$ & 0.000 & $-48,57$ & $-45,55$ \\
$\beta_{8}$ & 47,4 & 0,7078 & 66,9 & 0.000 & 45,87 & 48,89 \\
\hline \multicolumn{7}{c}{ Fonte: Pesquisa dos Autores (2012). }
\end{tabular}

Considerando os valores dos coeficientes, os dois fatores mais significativos para o modelo são o tipo de operador e a interação entre o tipo de operador e a duração do turno de trabalho.

Os menores custos ocorrem quando o turno tem cinco horas de duração e utiliza dois operadores experientes. Nesta situação os funcionários trabalhariam quase todo o tempo e teriam 
cerca de $100 \%$ de ocupação, algo impossível de ser conseguido em uma situação real. Além disso, nestes cenários, os funcionários trabalhariam menos de 8 horas diárias. Nesta condição, os funcionários estão legalmente impossibilitados de receber horas extras, algo muito restritivo para o processo, pois impediria o mesmo de lidar com imprevistos.

Diante destas considerações, uma alternativa mais viável e menos onerosa ocorre quando o turno se inicia às $17 \mathrm{~h}$, tem seis horas de duração e utiliza dois operadores experientes. Uma vez que nesta opção a taxa de ocupação dos funcionários possibilita certa margem de folga. Esta folga ajudaria a lidar com imprevistos no setor, uma vez que o tempo ocioso poderia ser eventualmente utilizado no processamento de CTRCs de pedidos que cheguem atrasados. Além disso, o grau de retrabalho e o tempo gasto pelos funcionários seriam reduzidos se este novo cenário proposto fosse implementado.

\section{CONSIDERAÇÕES FINAIS}

A abordagem proposta se contrapõe a abordagem tradicional de ensino da simulação e planejamento e análise de experimentos, no qual a ênfase está no domínio de softwares dedicados em detrimento do conhecimento e análise do próprio processo. Outro aspecto que merece destaque refere-se à utilização de planilhas eletrônicas para a realização da proposta apresentada. Se considerar a facilidade de acesso e a grande difusão das planilhas eletrônicas nas organizações, a abordagem utilizada pode ser vista como uma alternativa mais acessível do uso destas técnicas.

É importante frisar a sinergia do uso conjunto da simulação computacional, do planejamento e análise de experimentos e das técnicas de diagramação. Estas ferramentas possibilitam uma melhor compreensão do processo que será redesenhado. A sinergia destas ferramentas se faz presente pela complementaridade das mesmas, uma vez que a diagramação dos processos possibilita entender as características do processo, a modelagem computacional permite a criação de novos cenários e a visualização dos impactos dos diversos fatores no desempenho do processo. $\mathrm{O}$ planejamento e análise de experimentos, por sua vez, possibilita a seleção do melhor cenário.

Dada a importância da melhoria contínua do desempenho dos processos produtivos nas atividades conduzidas pelos gestores, técnicas voltadas para a análise e redesenho de processos são importantes conteúdos na formação dos Engenheiros de Produção. Diante disso, a estratégia proposta pode ser entendida como uma a alternativa didática para o ensino da simulação e do DOE em cursos de Engenharia de Produção.

\section{Abstract}

Given the importance of the use of discrete event simulation and Design of Experiments for the analysis and improvement of existing processes in organizations, this paper discusses the teaching 
of these techniques in the undergraduate course. This text proposes one teaching strategy whose emphasis is on understanding and analyzing the process to be simulated and optimized in contrast to existing approaches that focus on the knowledge of the specifics routines of the software dedicated to these techniques. The proposed approach is based on the work of Laguna and Marklund (2004), Hartvigsen (2001) and Mendenhall and Sincic (2012). The simulation is done using spreadsheets using the package SimQuick. The Design of Experiments for process optimization is done by using the techniques of ANOVA and Multiple Linear Regression. A case performed by a group of students is presented as a teaching tool to clarify the proposed teaching strategy.

Key-words:Process Analysis, Discrete Event Simulation, Optimization.

\section{Referências}

BARRENTINE, L. B. An Introduction to Design of Experiments: a simplified approach. Milwaukee: ASQ Quality Press, 1999.

BERENDS , P.; ROMME, G. Simulation as a Research Tool in Management Studies, European Management Journal, v. 17 , n. 6, p. 576-583, 1999. crossref

BORN, R. G.; STÅHL, I. A business course in simulation modeling, Issues in Information Systems, v. IX, n. 1, 2008.

HARTVIGSEN, D. SimQuick - process simulation with Excel. Second Edition, Pearson Pretince Hall, 2005.

HILL, R. R. Process Simulation in Excel for a Quantitative Management Course. INFORMS Transactions on Education, v. 2, n. 3, 2002. crossref

LAGUNA, M.; MARKLUND, J. Business Process Modeling, Simulation, and Design. New Jersey: Pearson Prentice Hall, 2004.

LEV, B. Book Reviews, Interfaces, v. 32, n. 5, 2002. crossref

MAHBOUBIANA, M. Educational aspects of business simulation softwares, Procedia Social and Behavioral Sciences, v. 2, p. 5403-5407, 2010. cross ref

MATHEUS, P. Design of Experiments with MINITAB. Milwaukee: ASQ Quality Press, 2005.

MEDINA, A. C.; CHWIF, L. Modelagem e Simulação de Eventos Discretos: teoria \& aplicações. $2^{\circ}$ Edição, São Paulo: Editora dos Autores, 2007.

MENDENHALL, W.; SINCICH, T. Second Course in Statistics - Regression Analysis. Prentice Hall, 7th Edition, 2012.

PYZDEK, T.;KELLER, P. Seis Sigma: Guia do Profissional. Rio de Janeiro: Alta Books, $2^{\circ}$ Edição, 2011.

SCHRAGE, M. Serious play: how the world's best companies simulate to innovate. Boston: Harvard Business School Press, 1999.

STÅHL, I. Teaching simulation to business students summary of 30 years’ experience, In. Proceedings of the 2007 Winter Simulation Conference, 2007. cross ref

VIEIRA, S. Análise de variância: Anova. São Paulo: Editora Atlas, 2006.

\section{Dados dos autores}

Nome completo: Noel Torres Júnior

Filiação institucional: Universidade Federal de Minas Gerais - UFMG

Departamento: Departamento de Ciências Administrativas 
Função ou cargo ocupado: Professor Adjunto

Endereço completo para correspondência (bairro, cidade, estado, país e CEP): Av. Antônio Carlos, 6627 - Pampulha - Belo Horizonte - MG - Brasil - CEP 31270-901

Telefones para contato: (31) 3409- 7316

e-mail: noelface@gmail.com / noel@face.ufmg.br

Nome completo: Júlia Zandomenico do Nascimento

Filiação institucional: Universidade Federal de Minas Gerais

Departamento: Engenharia de Produção

Função ou cargo ocupado: Estudante

Endereço completo para correspondência (bairro, cidade, estado, país e CEP): Rua Custódio de

Melo, 113 - Bairro Liberdade, Belo Horizonte, Minas Gerais, Brasil. CEP: 31270-790

Telefones para contato: (31) 9217-2012

e-mail: juliazn@globo.com

Nome completo: Guilherme Gonçalves de Souza

Filiação institucional: Universidade Federal de Minas Gerais

Departamento: Engenharia de Produção

Função ou cargo ocupado: Estudante

Endereço completo para correspondência (bairro, cidade, estado, país e CEP): Rua Vesta, 263,

Bairro João Pinheiro, Belo Horizonte, Minas Gerais, Brasil. CEP: 30530-500

Telefones para contato: (31) 9677-3152

e-mail: guilhermegoncalvesdesouza@gmail.com

Submetido em: 30/03/2013

Aceito em: 06/02/2014 